\title{
COMPARISON BETWEEN GOVERNMENT AND NON-GOVERNMENT WEBSITES OF NEPAL ON BACKLINKS AND BROKEN LINKS
}

\author{
Dinesh Bajracharya, \\ Kantipur College of Management and \\ Information Technology, \\ Tribhuvan University,
}

\begin{abstract}
Websites are the most effective and efficient medium for information distribution, communication, marketing and have the potential to enable new marketing trends. Links present in the pages allow you to easily access the information and navigate between the pages. Everybody likes websites with reliable, up-to-date, current content. Those websites will be linked by several other websites. A good, reliable, up-to-date website will have a decent number of backlinks. A wellmanaged website always helps users with its content and be of value for the users. But websites with stale content, broken links (dead links) will become annoyance. The value of links has often been taken for granted because there is no clear measure of their worth. The reason for this is simply the fact that links are everywhere. Links are essentially the threads that hold the whole World Wide Web together.
\end{abstract}

This paper experiments, examines and compares the number of broken links and backlinks in the government and non-government web pages of Nepal. Online Broken Link Checker and Backlink Checkers were used to study the sample websites.

Keywords: Backlinks, Broken links, Government, nongovernment, website, Nepal.

\section{INTRODUCTION}

Several organizations either government or nongovernment are established with the view of servicing people (users), they used to have different kinds of services, products or information valuable for the people. These organizations disseminate information about the services, products and information using

\author{
Yunisha Bajracharya, \\ Islington College, \\ London Metropolitian University
}

different media like newspaper, websites, social media, televisions.

Organizations use different media to provide information about themselves and services to the people, but their own websites are the most cost effective and efficient one for information dissemination. Those websites can be accessed by people from anywhere anytime and get information.

It is necessary for any organization to make their website up to date with accurate, fresh and reliable content, there should not be any kind of issues with the web pages. Websites with good content will be liked by lots of people and external organizations; external organizations may authorize them and may also put link in their website (backlink). Websites with stale, inaccurate contents and links pointing to nowhere (broken links) are just annoyance to the users and will not be liked by most of the users [8]. This research is conducted to understand which type of website: the government or nongovernment website of Nepal has more backlinks and broken links; which organization is more responsible in maintaining the website.

Desk research method was used for this study, a list of websites (urls) to evaluate was prepared, online tools for backlink and broken analysis were searched in google, each url from the list was supplied to the online tools and results were tabulated in excel sheet for analysis.

\section{Research question}

While analyzing the government and non-government websites, we focused on following research questions.

1. Which type of organization (government or nongovernment) has more backlinks and more broken links?

2. Are the government and non-government offices aware about the broken links in their websites? 


\section{International Journal of Engineering Applied Sciences and Technology, 2020 Vol. 5, Issue 2, ISSN No. 2455-2143, Pages 98-104 \\ Published Online June 2020 in IJEAST (http://www.ijeast.com)}

3. Is there impact of broken links in SEO of a website?

\section{RELATED STUDIES}

There have been a few review articles on broken links, backlinks and its impact on website ranking. The paper, composed by Christos Ziakis, Maro Vlachopoulou, Theodosios Kyrkoudis, and Makrina Karagkiozidou titled included Important Factors for improving Google Search Rank. Further identified the principle factors influencing the ranking of websites [3]. They explained that Backlinks play a major impact in ranking websites. Google, has developed an algorithm called "Pagerank" which calculates the relevance and significance of the webpages. The positioning of a site highly depends on the Pagerank calculation and is the only default factor for website rankings. Due to this reason, we need to have quality backlinks that reference the heavily traffic websites, as it is crucial to achieve better ranking.

On similar papers published by Meenu Dave on The Role of Backlinks in Search Engine Ranking [1], mentioned that the quality and quantity of backlinks from relevant sites is one of the most important factors for optimal website ranking. The number of backlinks used with relevant keywords impact in SEO. J. Varadharajal, Dhanavan have analyzed web impact factor (WIF) for the websites of state universities in Kerala. According to them, the ratio of connections made in the site determines the quality of pages of the site [6]. Furthermore, they also suggested that outside links are more valuable for a website.

The paper written by Sarah Glasser on Broken Links and Failed Access: How KBART, IOTA, and PIE-J Can Help [14] mentioned the ways to improve the web content. She researched on link resolver tools that analyses open URLs and found that $20 \%$ of the fulltext link were inaccurate. Some of the tools showed incorrect convenience data (false positives). The reason was found to be due to the broken links in the websites that changed the results to false positive, which in turn damaged the user experience [14]. Web surfers do not prefer broken links, broken links are just annoyance for the surfer, web surfer usually tend to avoid sites with many broken links [8].

Similarly, on an article published on "ScienceDirect" by Shariq Bashir titled Updating broken web links: An automatic recommendation system on April 2019 researched on the contextual information portals (CIP). This innovative technology focused on repairing the broken links by developing a system called brLinkRepair. This system generates a list for each broken link and their pointed websites. For each broken link, brLinkRepair recommends a related page that is similar to the pointed page of broken link through the query generation mechanism to search related pages [2].

\section{THEORETICAL FOUNDATION}

Websites have become an easy tool for organizations to provide information about services and products provided by them, and to communicate with the users. Websites can contain data in different formats (text, audio, video, images etc.). This capability of websites has helped the organizations to disseminate information to the public quite easily. Also, it is easy for the public to get information anytime from anywhere.

A website is just a collection of documents or files that can be accessed through the internet and may have links to other external web pages [9]. It can be defined as the assortment of interconnected web pages, multimedia contents that are identified by unique domain name and published in a web server. Pages of websites are interconnected by a special tag called anchor $(\langle\mathrm{a}\rangle)$ and its HREF property. These pages are viewed using a program called a browser. The most popular browsers are Chrome, Firefox, Internet Explorer and Safari.

Referring Domain is the domain of website which is referring back to the search domain and Referring IP is the IP address of the website which backlinks to another website [10].

Users use search engines to search information/data using search texts. Search engines are software which takes texts from users and replies users with several pages related to the user's search text. Those pages are displayed in order, that is each page is given rank by the search engine. Search engines have page rank algorithm or formula, the page ranking algorithm ranks web pages using rules; pages shown in the initial ranking of the search engine are considered as more relevant and are usually viewed by users [5].

But despite whatever formula is used to rank pages, the goal is to connect the searcher with the information. Search engines rank websites/pages according to the value of the site or page, and sites with high rank will be displayed in the first page of the search engine. So, it is necessary for organizations to make search engine friendly web pages.

There are several factors that play a role in the ranking of the website/web pages. But it is the content which must be very good. Some of the major factors 
influencing the ranking of websites are Content, Design and Backlinks.

Making websites reliable with up-to-date content is the responsibility of the management and IT department of the organization (co-adaptation between information officers and managers). The team should check their contents, and provide error free, reliable fresh information. Matching the user's query with the solution and type of content determines the quality of the website [13].They also need to keep track of the popularity of their website and monitor the user traffic details like how many users are accessing it, from which locations, at what time and so forth. The management should make note of how many other websites are linking to their website or if there are any dead link or broken links (links which when clicked to result nothing or 404 error in their website). Broken links do not have a direct impact on the SEO of the website, but users will avoid sites with broken links, number of visitors will decrease, this will influence the ranking of the site in search engine. Cleaning up these broken links can add to the content quality, thus resulting in better user experience and easier discovery for search engines [13].

Government organizations considered for this study are primarily service providing organizations. These organizations are governed by the government of Nepal and operate under the rule and regulations of the Nepal government. Non-government organizations considered for this study are also service providing organizations governed by a limited number of people who have invested in the organization. These organizations have developed their own set of rules and regulations to operate the organization and these organizations are not above the law of Nepal. These both kinds of organizations are directly concerned with the public service, the people come to the organization to get services provided.

These organizations provide notices and information about their services through different media, and website is one of them. It is very necessary for these organizations to have UpToDate website with fresh and accurate contents. Useless links and broken links in these websites are just annoyances for the public, the public need fresh and accurate content. Also, these websites' content must be of value for other organizations, should have a very good number of backlinks. These websites must be trustworthy [11]. The importance of backlinks and annoyances from broken links have been studied by several authors but rarely in combination of both. Backlinks are references from relevant sites that highlight the page. Having backlinks from high ranked pages increases the ranking of the page. The organization or the company alone is responsible for maintaining the quality of these factors so cannot be avoided while building the websites.

Christos Ziakis [3] has conducted research to find out factors to improve Google Search Rank. They have used three samples of websites, where the first 15 search results were extracted. They used Google to study the websites and impact of considered factors. The factors considered were SSL, keywords, quality and quantity of backlinks and structure. Through this study, they found that internal linking is an important factor for individual pages of a website. The internal linking not only provides good user experience but also facilitate tracing and indexing of individual components of the website.

The value given by external links (backlinks) should be shared uniformly in all subpages of the website to get higher ranking. It is necessary to check internal links regularly if it is broken or not. The quality and quantity of backlinks is one of the most important factors for achieving higher rank. So, it is necessary to build good backlinks that are relevant to the linked sites topic.

J. Varadharajul and Dr. S. Dhanavandan have done analysis of Web Impact Factor (WIF) for the websites of State Universities in Kerala. They have used a list of state government universities in Kerala provided by UGC India, thirteen universities were considered for the study. Their objective was to examine WIF for websites of State Universities of Kerala. Data collection was done using a Web ranking tool. Their research has found that no links to institutional research material, unawareness of web ranking by webmasters and university web developers, structural problems on web design, limitation of access to material, absence and non-linkage of institutional repositories as some of the factors contributing to increase or decrease the impact of academic website globally [6].

\section{METHODOLOGY}

Desk Research was performed for this study. Desk research is the collection of secondary data from internal sources and is frequently carried out without fieldwork. A list of organizations and their websites about which study was to be conducted was prepared. Each website was tested in the online backlink checkers and broken link checker tools. Existing free online tools were used to evaluate broken links and backlinks in websites.

Data generated for each website about backlinks and broken links were tabulated. Tabulated data were 
analyzed for the comparative study between government and non-government organization websites.

\section{RESEARCH DESIGN}

\section{Data Collection and analysis}

During our research, we focused on a list of websites to evaluate for backlinks and broken links. For the evaluation we searched for online free tools in google using "Broken link checker" and "Backlink finder" texts and considered www.brokenlinkcheck.com and www.seoreviewtools.com for the analysis purpose.

i. www.brokenlinkcheck.com: This is an online link checker tool that allows users to check their entire website for broken links and helps you identify exactly where to fix. For every invalid webpage reference, this link checker displays the page source and highlights the actual HTML code that contains the broken link; thus, helping the developers to easily fix their mistakes and eradicate the PAGE NOT FOUND errors.

ii. www.seoreviewtools.com: This is an online real-time SEO tool that combines Search Engine Optimization \& Web development. This tool analyses the user input websites based on 15 various SEO checks.
The listed URLs of websites were fed one by one to the chosen online tools and results displayed for site were copied to excel sheets. The mean and standard deviation for both broken links and backlinks were calculated, and student t-test was performed to compare the significant difference between the number of broken links and backlinks between government and nongovernment websites.

\section{Results and Discussion}

The output given by the online tools were stored in the spreadsheets. The table contains external backlinks, follow links, domain referring, referring IPs, and broken links for each of the website.

Average backlinks and broken links and standard deviation of links in each type of website was calculated. The results of calculations are presented in the following tables.

\section{Broken link analysis}

To know which type of organization has more broken links, mean and standard deviation in the website were calculated for each type of websites. The calculated mean and standard deviations are shown in following table.

Table 1. Number of broken links, backlinks, referring domains, referring IPs of considered websites

\begin{tabular}{|c|c|c|c|c|c|c|}
\hline SNo. & code for org & $\begin{array}{l}\text { External } \\
\text { Backlinks }\end{array}$ & $\begin{array}{l}\text { Follow } \\
\text { Links }(\%)\end{array}$ & $\begin{array}{l}\text { Domain } \\
\text { Referring }\end{array}$ & $\begin{array}{l}\text { Referring } \\
\text { IPs }\end{array}$ & broken links \\
\hline 1 & gov_ws_1 & 132 & 100 & 5 & 5 & 13 \\
\hline 2 & gov_ws_2 & 150 & 33 & 6 & 6 & 23 \\
\hline 3 & gov_ws_3 & 242 & 75 & 26 & 19 & 0 \\
\hline 4 & gov_ws_4 & 422 & 64 & 40 & 41 & 0 \\
\hline 5 & gov_ws_5 & 819 & 56 & 44 & 53 & 5 \\
\hline 6 & gov_ws_6 & 1307 & 83 & 71 & 76 & 3 \\
\hline 7 & gov_ws_7 & 2893 & 71 & 506 & 237 & 4 \\
\hline 8 & gov_ws_8 & 3686 & 88 & 115 & 96 & 21 \\
\hline 9 & gov_ws_9 & 4310 & 55 & 283 & 218 & 20 \\
\hline 10 & gov_ws_10 & 4429 & 88 & 58 & 69 & 10 \\
\hline 11 & gov_ws_11 & 4824 & 83 & 55 & 60 & 1 \\
\hline 12 & gov_ws_12 & 5029 & 77 & 582 & 475 & 9 \\
\hline 13 & gov_ws_13 & 7752 & 81 & 255 & 202 & 23 \\
\hline
\end{tabular}


International Journal of Engineering Applied Sciences and Technology, 2020

Vol. 5, Issue 2, ISSN No. 2455-2143, Pages 98-104

Published Online June 2020 in IJEAST (http://www.ijeast.com)

\begin{tabular}{|l|l|r|r|r|r|r|}
14 & gov_ws_14 & 7951 & 71 & 149 & 87 & 22 \\
\hline 15 & gov_ws_15 & 8603 & 51 & 1210 & 934 & 27 \\
\hline 16 & ngov_ws_1 & 5 & 67 & 3 & 3 & 1 \\
\hline 17 & ngov_ws_2 & 24 & 33 & 9 & 8 & 4 \\
\hline 18 & ngov_ws_3 & 25 & 82 & 11 & 7 & 4 \\
\hline 4 & ngov_ws_4 & 60 & 100 & 13 & 12 & 5 \\
\hline 5 & ngov_ws_5 & 62 & 92 & 25 & 25 & 1 \\
\hline 6 & ngov_ws_6 & 217 & 69 & 26 & 36 & 20 \\
\hline 7 & ngov_ws_7 & 271 & 74 & 74 & 71 & 11 \\
\hline 8 & ngov_ws_8 & 1280 & 35 & 39 & 42 & 1 \\
\hline 9 & ngov_ws_9 & 1285 & 48 & 132 & 136 & 10 \\
\hline 10 & ngov_ws_10 & 1310 & 64 & 29 & 36 & 6 \\
\hline 11 & ngov_ws_11 & 1380 & 57 & 7 & 7 & 10 \\
\hline 12 & ngov_ws_12 & 3636 & 73 & 79 & 88 & 3 \\
\hline 13 & ngov_ws_13 & 4387 & 60 & 23 & 192 & 1 \\
\hline 14 & ngov_ws_14 & 5093 & 49 & 225 & 190 & 36 \\
\hline 15 & ngov_ws_15 & 5536 & 73 & 223 & 208 & 14 \\
\hline
\end{tabular}

Table 2. mean and standard deviation of broken links for government and non-government websites

\begin{tabular}{|l|l|l|}
\hline & Gov & non-gov \\
\hline Mean & 12.06667 & 8.466667 \\
\hline standard deviation & 9.742885 & 9.425396 \\
\hline
\end{tabular}

Table 2 shows that the mean of broken links for government websites is higher than for the nongovernment websites; the is a gap of more than 3.5 units. The standard deviation for the government websites is less than mean but this is not the case for non-government websites. This suggests that there is a more variation in the number of broken links for nongovernment websites compared to the government websites.

Further to know if there is a significant difference between the number of broken links between considered types of organizations, student-t test was performed at the $5 \%$ level of significance for two-tail test. The result of t-test showed that the average number of broken links in government websites are significantly different from the average number of broken links in the non-government websites at 5\% level of significance.

\section{Backlink analysis}

To know the content of which type of organization is considered reliable, mean of backlinks and standard deviation of backlinks between considered types of organizations are calculated and tabulated in the following table.

Table 3. mean and standard deviation of backlinks for government and non-government websites

\begin{tabular}{|l|l|l|}
\hline & Gov & non-gov \\
\hline Mean & 3503.267 & 1638.067 \\
\hline standard deviation & 2983.238 & 1997.187 \\
\hline
\end{tabular}

A big difference between the mean number of backlinks between government and non-government websites was seen from table 3. Government websites have got more backlinks than non-government websites, this suggests that the content of government websites are better and authentic than that of nongovernment websites.

The standard deviation for the government websites is less than its mean but, here also, the standard deviation for non-government websites are higher than its mean. This suggests that some of the non-government 


\section{International Journal of Engineering Applied Sciences and Technology, 2020 \\ Vol. 5, Issue 2, ISSN No. 2455-2143, Pages 98-104 \\ Published Online June 2020 in IJEAST (http://www.ijeast.com)}

websites have got very high backlinks and some have got very less.

Student t-test was performed to see if there is significant difference between the average number of backlinks between government and non-government websites. The result showed that there is a significant difference between the mean number of backlinks between two types of website at the $5 \%$ level of significance.

\section{DISCUSSION AND SUGGESTIONS}

It was found that the average number of broken links and backlinks between two types of organizations (government and non-government) are significantly different. Websites of government organizations are getting more backlinks and broken links than nongovernment organizations. This suggests that lots of external websites are finding government websites informative and good.

At the same time government organizations' websites are having more broken links. From this we can derive following conclusions:

- Government organizations websites are more authentic than nongovernment websites

- Government organization are not aware of broken links and impact of broken links over the users

A big variation is seen in the number of broken links and backlinks for the non-government organizations. Some of the non-government organizations have a very good number of backlinks and some have very few, also some have many broken links and some have very less number.

Broken links are not pleasing to users, it is just annoyance, waste of time of users. As users find more and more broken links in the website, they will start to search for other websites where they can find data/information they were searching for. This will decrease the number of visitors of the website and will indirectly impact the ranking in search engine.

So, it is necessary for organizations to take good care of their website, website contents, links. If contents are out-of-date, or links are broken, then those issues must be immediately corrected. The proper management of website and contents may result in winning trust of viewers and increasing in ranking of the website.

Backlinks are very important for any website. Backlinking a website $\mathrm{A}$ by another website $\mathrm{B}$ is a kind of endorsement to the website A by B, B is outsider for A. Website B believed that the content of A is reliable, up-to-date and will of value for viewers. This will help to increase the number of viewers for A also.
Viewers hesitate to visit and backlink websites with stale content and broken links, the decrease in backlinks and viewer because of bad content result in decline popularity of website and decline in rank of the website.

Government organizations supposed to provide authentic, reliable and up-to-date content, they must be aware about any kind of issues and problem with their websites. But sometimes government organizations are seemed to ignorant at presenting important information online, not paying enough attention for the peoples' information need. Broken links, stale contents and mysterious abbreviations are some of the problem seen in the government websites [7].

A greater number of broken links was found in government websites compared to non-government websites; this shows some kind of reluctance in maintenance of their websites is seen in government websites.

Backlinks are an important factor for the popularity of any website. Organizations can increase number of backlinks to their websites using following strategies [1]:

- Organizations can submit the URL of their websites to reputed online Directories.

- Use blogging sites to promote their websites, that is writing comments, articles about the website, content of websites and publishing in blogs.

- Creating bookmarks in social media sites like Facebook, Twitter, Linked etc.

- Finding broken links in other's websites and informing about the broken links to that organization, that is doing a favor to others, in return other websites may put backlink to information provider.

- Building internal links without any problem, correcting broken links; this will increase navigability of the website and also user experience of navigating website.

- Promoting content using other media like radio, newspaper etc.

A broken link may not seem to be so bad on the surface, but it can do some serious damage indirectly to the website and the reputation of the organization. The ranking of the website may be hurt. Users get frustrated by finding broken links in the website and get something annoying instead of searching for information, their experience of browsing the website will not be good and results in loss of user and revenue as well. Around $88 \%$ of users are reluctant to come back to a site after a bad experience [4].

Broken links can be corrected or minimized using following strategies [12]:

- Creating links to primary stable sources rather than secondary sources 


\section{International Journal of Engineering Applied Sciences and Technology, 2020 \\ Vol. 5, Issue 2, ISSN No. 2455-2143, Pages 98-104 \\ Published Online June 2020 in IJEAST (http://www.ijeast.com)}

- Avoiding links that point to another web page in the site

- Using persistent identifiers

- Avoiding links to documents

- Avoiding deep links

- Broken links can be redirected to new location using redirection mechanisms

- Using software to check for broken link and Automatically correcting broken link to point new URLs

- $\quad$ Finding broken links and manually correcting those links.

\section{CONCLUSION}

Government websites are found more authentic compared to non-government websites. However, government websites have more broken links than non-government websites, suggesting lack of maintenance of website, one of the most influential and cost-effective tools for information dissemination, communication and interaction, in the part of government organizations compared to nongovernment organizations. There is indirect connection between broken links and backlinks of the website. Broken links in a website indirectly impact rank of the website and the possibility of getting more backlinks by high ranked website will also decrease. So, it is necessary for both types of organization to take good care of their websites, keep websites up-todate and broken link free to achieve a greater number of viewers and backlinks. Government websites are found to have more backlinks than non-government websites. This suggests that government websites are more authentic, and useful even having more broken links.

\section{REFERENCES}

[1] Ayush Jain, M. D., 2013. The Role of Backlinks in Search Engine Ranking. International Journal of Advanced Research in Compuer Science and Software Engineering, 3(4), pp. 596-599.

[2] Bashir, S., 2013. Broken Link repairing system for constructing contextual information portals. Journal of King Saud University - Computer and Information Sciences, 31(2), pp. 147-160.

[3] Ziakis C., M. V. T. K. a. M. K., 2019. Important Factors for Improving Google Search Rank. Future Internet, 11(2).

[4] Gavril, A., 2018. Why broken links can hurt your website and what to do about them. [Online]
Available at: https://www.123-reg.co.uk/blog/seo2/broken-links-can-hurt-website/

[Accessed 19 Jan 2020].

[5] Goodwin, D., 2018. What is SEO? Here's Search Engine optimization Defined by 60 experts. [Online] Available at:

https://www.searchenginejournal.com/seo-101/whatis-seo/

[Accessed 27 Dec 2019].

[6] Varadharajalu J., Dhanavandan S., 2017. Analysis of Web Impact Factor (WIF) for the Websites of State Universities of Kerala. [Online]

[Accessed 29 December 2019].

[7] Barret K., R. G., 2017. Why are Government Websites so bad?. [Online]

Available at:

https://www.governing.com/columns/smartmgmt/gov-legislative-websites-problems.html [Accessed 19 Jan 2020].

[8] Lal, A. K., 2010. Protocol to Fix Broken Links on the World Wide Web. United States Patent.

[9] Jalal S. K., S. C. B. P. M., 2010. Web Impact factor and link analysis of selected India Universities. Annal of Library and Information Studies, Volume 57, pp. 109-121.

[10] Semrush, 2019. Backlinks Referring Domains \& Referring IPs Reports. [Online]

Available at: https://www.semrush.com/kb/503backlinks-referring-domains-report-manual [Accessed 31 December 2019].

[11] Soulo, T., 2018. Introducing the NEW Ahrefs' Domain Rating (and how to use it). [Online] Available at: https://ahrefs.com/blog/domain-rating/ [Accessed 28 December 2019].

[12] Vertexplus, 2016. Why Broken Links Are Bad For Business. [Online]

Available at: https://medium.com/@vertexplus/whybroken-links-are-bad-for-business-6dfe8477573d [Accessed 30 Dec 2019].

[13] Stox P., 2016, Broken link building made easy, [Online] Available at:

"https://searchengineland.com/broken-link-buildingmade-easy-269137?curator=MediaREDEF", [Accessed 21 March 2020]

[14] Glasser S., 2012, Broken Links and Failed Access: How KBART, IOTA, and PIE-J Can Help, ALCTS, Vol 56, No 1. 